\title{
Flow and particles deposition in anatomically realistic airways
}

\author{
T. XIONG ${ }^{\mathrm{a}}$ and H. ILMI ${ }^{\mathrm{a}}$ and Y. HOARAU*a and P. CHOQUET ${ }^{\mathrm{b}}$ and C. GOETZ ${ }^{\mathrm{b}}$ \\ and A. FOURAS ${ }^{\mathrm{c}}$ and S. DUBSKY ${ }^{\mathrm{c}}$ \\ and M. BRAZA ${ }^{\mathrm{d}}$ and S. SAINLOS-BRILLAC ${ }^{\mathrm{d}}$ and F. PLOURABOUÉ ${ }^{\mathrm{d}}$ and D. LO JACONO ${ }^{\mathrm{d}}$ \\ ${ }^{a}$ IMFS, 2 rue Boussingault, Strasbourg, France \\ ${ }^{b}$ Imagerie Préclinique des Hôpitaux Universitaires de Strasbourg, France \\ ${ }^{\mathrm{c}}$ Laboratory of dynamic imaging, Monash University, Australia \\ ${ }^{d}$ IMFT, av. du Pr. Camille Soula, Toulouse, France
}

\section{Introduction}

Keywords: particle deposition; rat lungs; rabbit lungs; numerical simulations
The aerosol particles present in our environment are identified as increasingly risk factors for the human health. In particular, small particles that reach the surface of gas exchange in the alveolar region of lungs are considered most harmful to health. Nevertheless, aerosols can also be used for diagnostic or therapeutic purposes to treat lung diseases. Inhalers, for example, are commonly used to administer drugs. In cases of lung disease, administration of medication by aerosol has the advantage of reaching the region directly affected and requires lower doses (side effects are minimized).

The morphological descriptions of the bronchial tubes of the man appeared in the literature since 1963, when Weibel [1] introduced a physical symmetric model. The human lung is a sequence of bifurcations in two parts. It has an average of 23 generations of bifurcations which represents about 17 million of bifurcations. The last 5 generations are covered with cellular structures through which the exchange takes place with the blood gas [2].

The understanding of the flow structures and the particle transport/deposition across the human bronchial system remains a challenge to achieve because of the complexity of the geometry of human lungs.

This work relies a strong collaboration between physicians, medical imaging researchers, fluid mechanics researcher and CFD researchers.

\section{Methods}

Our study focuses on the numerical modeling of the flow and particles deposition in airways using the commercial package $C F D-A C E$. The objective of this study is to propose a reliable and complete numerical model of transport of particles in the pulmonary flow by taking into account the full breathing cycle. For the moment the movement of the airways imposed by the breathing are not taken into account.

*Corresponding author. Email: hoarau@unistra.fr
This study is divided into four parts :

- $\quad$ First the CFD-ACE package is validated regarding flow $[3,4]$ and particles deposition in a simple model $[5,6]$

- Simple geometries derived from the Weibel model are studied

- An anatomically realistic model of rat lung is simulated

- A more complex geometry of rabbit lung is simulated

The particles trajectories and deposition are obtained by solving a lagrangian transport equation where only the drag force is retained. The deposition efficiency is computed as the ratio of the amount of particles sticked at the wall over the total number of released particles.

The rat geometry is obtained after an adult rat killed by IV administration of barbiturics, was infused with a iodine contrast agent $(10 \% \mathrm{v} / \mathrm{v}$, Visipaque320, GE Healthcare, Chalfont St Giles, UK). Imaging of the thorax was performed ex vivo using an X-Ray $\mu$ CT scanner (eXplore Vision 120, GE Healthcare, Waukesha, USA). The protocol used involved 360 views over $360^{\circ}$, with 1 frame averages, at $100 \mathrm{kV}, 50 \mathrm{~mA}$. After reconstruction using a Feldkamp algorithm of backprojection, the imaging volume was made of cubic voxels of $49 \mu \mathrm{m}$ x $49 \mu \mathrm{m}$ x $49 \mu \mathrm{m}$. Visualisation was performed using MicroView (GE Healthcare, Waukesha, USA). After invertion of grey levels, a surface rendering (marching cubes algorithm) was applied after thresholding. The surface was then exported in stl format and prepared in gmsh before being meshed in CFD-GEOM.

The rabbit geometry was obtained using synchrotron-based dynamic computed tomography. Details of the experimental technique and segmentation protocol are provided elsewhere [7]. Briefly, newborn rabbit pups are ventilated and imaged using propagation-based phase-contrast imaging. The lungs are an ideal sample for phasecontrast imaging, as they exhibit large phase boundaries at air-tissue interfaces. 
This technology can provide rapid imaging of the lungs without contrast agent [8]. By synchronising the image acquisition with the ventilation and rotating the sample, four-dimensional ( $4 \mathrm{D}=3 \mathrm{D}+$ time) images of the bronchial tree can be achieved. Again, one stl geometry was prepared in gmsh before being meshed in CFD-GEOM.

Most of this work was performed during $\mathrm{H}$. Ilmi $\mathrm{PhD}$ thesis [9], the remaining part was studied by $\mathrm{T}$. Xiong during her master 2 internship.

\section{Results and Discussion}

Concerning the Weibel's generic models, the solutions generated by the solver CFD-ACE were in very good agreement with the literature and we evidenced the importance of the Dean vorticies on both the flow and the particles deposition. The deposition is essentially of inertial type and the deposition efficiency increases with both the Stokes number and the Reynolds number. The deposition occurs mainly at the bifurcations. We have simulated different geometries where the successive bifurcations were rotated by $90^{\circ}$ and we observed a strong influence of the geometry : at the first bifurcation the Dean vorticies are properly splitted in each bifurcation and the flow rate in the inner tube is much higher but at the second bifurcation the Dean vorticies are broken and the flow is more chaotic, the particle deposition is also significantly higher.

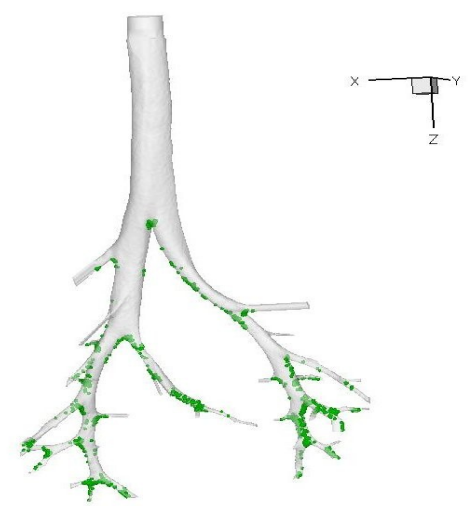

Fig. 1 : deposition in rat lungs

The simulations of the rat and the rabbit lungs raised the major difficulty of this study : the appropriate grid generation. Very fine grids were necessary to obtain grid convergence on the particles deposition and such grids is very difficult to obtain in this kind of complex geometries.

The rat geometry is quite linear and no recirculations were found. The particles deposition is again mainly inertial, increases with the Stokes number and occurs in the lower bifurcations (Fig. 1 ) because they are pushed toward the wall after each bifurcation.

The rabbit geometry is much more complex and a large and strong vortex appear near the inlet because of the intubation which causes abnormal particle deposition at the inlet (Fig. 2). Dean vorticies were evidenced in few bifurcations. The deposition occurs at the bifurcations in the lower part of the airway and again it increases with the Stokes number which is characteristic of the inertial process.
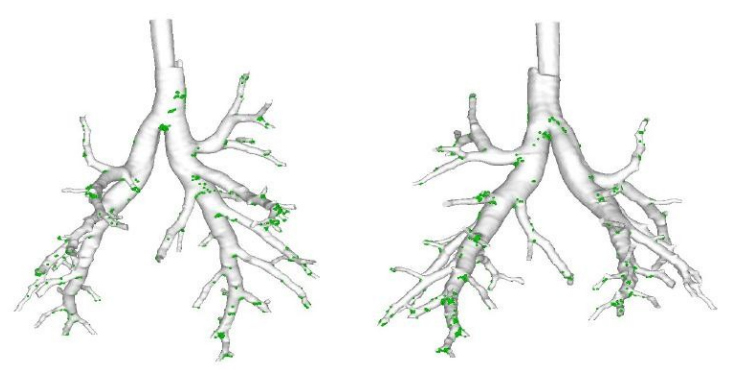

Fig. 2 : deposition in rabbit lungs

\section{Conclusions}

After a validation phase, we have simulated the flow and the particle deposition in many Weibel's generic models and in anatomically realistic geometries of rat and rabbit coming from medical imaging. The particle deposition is mainly inertial and increases with the Stokes number and the Reynolds number. One of the major difficulty of this study is the generation of the appropriate mesh for the realistic geometries.

\section{References}

[1] E. R. Weibel, Morphometry of the human lung. New York, Academic Press, 1963.

[2] E. R. Weibel, The pathway for oxygen, structure and function in the mammalian respiratory system. Harvard University Press, 1984

[3] Y. Zhao and B. B. Lieber. Steady inspiratory flow in a model symmetric bifurcation. Trans. ASME: J. Biomech. Engrg., 116:488-496, 1994

[4] J. K. Comer, C. Kleinstreuer, and Z. Zhang. Flow structures and particle deposition patterns in double bifurcation airway models, part 1 . air flow fields. Journal of Fluid Mechanics, 435:25-54, 2001

[5] C. S. Kim and D. M. Fisher. Deposition characteristics of aerosol particles in sequentially bifurcating airway models. Aerosol Science and Technology, 31:198-220, 1999.

[6] J. K. Comer, C. Kleinstreuer, and C. S. Kim. Flow structures and particle deposition patterns in double bifurcation airway models. part 2. aerosol transport and deposition. Journal of Fluid Mechanics, 435:55-80, 2001.

[7] Dubsky, S., Hooper, S.B., Siu, K.K.W. \& Fouras, A.(2012) Synchrotron-based dynamic computed tomography of tissue motion for regional lung function measurement. Journal of the Royal Society Interface. (Published online: 12/03/2012)

[8] Fouras, A., Allison, B.J., Kitchen, M.J., Dubsky, S.,Nguyen, J.T., Hourigan, K., Siu, K.K.W., Uesugi, K., Yagi, N., Lewis, R.A., Wallace, M.J. \& Hooper, S.B. (2012) Altered lung motion is a sensitive indicator of regional lung disease. Annals of Biomedical Engineering,40(5), 1160-1169.

[9] H. Ilmi, Modélisation numérique des écoulements pulmonaires. Thèse de l'Université de Strasbourg, 2012 